\title{
Quantitative PET Comparing Gated with Nongated Acquisitions Using a NEMA Phantom with Respiratory-Simulated Motion
}

\author{
Douglass C. Vines ${ }^{1,2}$, Harald Keller ${ }^{1,2}$, Jeremy D.P. Hoisak ${ }^{3}$, and Stephen L. Breen ${ }^{1,2}$ \\ ${ }^{I}$ Radiation Physics, Princess Margaret Hospital, Toronto, Ontario, Canada; ${ }^{2}$ Department of Radiation Oncology, University of Toronto, \\ Toronto, Ontario, Canada; and ${ }^{3}$ Department of Medical Biophysics, University of Toronto, Toronto, Ontario, Canada
}

\begin{abstract}
This study evaluated the use of gated versus nongated PET acquisitions for absolute quantification of radioisotope concentration (RC) in a respiratory motion-simulated moving phantom filled with radioactive spheres and background for both 2-dimensional (2D) and 3-dimensional (3D) acquisitions. Methods: An imagequality phantom with all 6 spheres filled with the same ${ }^{18} \mathrm{~F} \mathrm{RC}$ (range, 19-62 kBq/mL) was scanned with PET/CT at rest and in motion with and without gating. The background was filled with ${ }^{18} \mathrm{~F}$ solution to yield sphere-to-background ratios of approximately $5,10,15$, and 20 to 1 . Both $2 \mathrm{D}$ and $3 \mathrm{D}$ acquisitions were used for all combinations. Respiratory motion was simulated by using a motor-driven plastic platform to move the phantom periodically with a displacement of $2 \mathrm{~cm}$ and a cycle time of $5.8 \mathrm{~s}$. For gated acquisitions, the phantom was tracked using a real-time position management system. Images were reconstructed, and regions of interest with the same sizes as the actual spheres were manually placed on axial slices to determine maximum and mean pixel RC. A threshold method $(70 \%$ and $94 \%$ for $2 \mathrm{D}$ and $3 \mathrm{D}$ modes) was also used to determine a mean voxel RC. All values were compared with the expected RC; percentage differences were calculated for each sphere. To reduce partial-volume effects, only data for the 4 largest spheres were analyzed. Results: The mean pixel method was the only method with linear responses for all 3 scan types, enabling direct comparisons. The ranges of $\mathrm{RC}$ percentage differences were underestimated for all scan types (using the mean pixel method). The overall mean percentage differences were 37, 49, and 41 in 2D mode and 40, 51 , and 41 in 3D mode for static, nongated, and gated acquisitions, respectively. Gated acquisitions improved quantification (by reducing underestimation) over nongated acquisitions by $8 \%$ and $10 \%$ for 2D and 3D modes. Conclusion: In the presence of motion, the use of gated PET acquisitions appears to improve quantification accuracy over nongated acquisitions, almost restoring the results to those observed when the phantom is static.
\end{abstract}

Key Words: quantitative PET; gated PET; 4D PET; respiratory gating; NEMA phantom

J Nucl Med Technol 2007; 35:246-251

DOI: $10.2967 /$ jnmt.107.040782

\footnotetext{
Received Feb. 15, 2007; revision accepted May 15, 2007.

For correspondence or reprints contact: Douglass C. Vines, CNMT, PET, Princess Margaret Hospital, Radiation Physics, Room 5-612, 610 University Ave., Toronto, Ontario, Canada, M5G 2 M9.

E-mail: doug.vines@rmp.uhn.on.ca

COPYRIGHT @ 2007 by the Society of Nuclear Medicine, Inc.
}

$Q$ uantification of PET data is important in staging disease, monitoring disease status, and assessing treatment response (1-4). The presence of respiratory motion during PET can cause blurring of the moving objects in the image. This blurring can reduce the detection and image quality of lesions by decreasing lesion contrast, which can lead to a loss in the quantitative accuracy of radioactivity in the lesions (5-8).

The gating of PET acquisitions to respiratory motion is similar to cardiac gating and has been used to reduce the degrading effects of motion (5-7). Motion-gated PET requires the acquisition of many motion cycles over time and the division of the cycles into several equal time bins. The data for each bin of the cycle are stored separately, and at the end of 1 cycle the process begins again. Hence, within a single bin, motion blurring is reduced, and the series of bins corresponding to different time points throughout the motion cycle reduces blur in the entire cycle.

Respiratory-gated PET has been performed in various patient studies at different institutions (5-7,9-12). However, to evaluate the effectiveness of gating on PET, one needs to know the true radioactivity distribution and amount in the object being imaged without motion. A phantom with a known radioisotope distribution can be imaged first without motion (this is the gold standard) and then again in the presence of known motion to model the effects of motion. With a phantom, the parameters of motion such as direction, displacement, and cycle time can be known and controlled. Of the published phantom studies, some have been performed to evaluate the effects of motion on radioisotope quantitation $(7,8)$, whereas others have evaluated the effectiveness of gated PET $(5,6,10,11)$. Some studies have used high levels of radioisotope solutions exceeding clinical levels $(7,10,11)$, and all have used only 1 target-to-background radioisotope concentration $(\mathrm{RC})$ ratio. All have evaluated motion in only 1 direction.

In this study, we used a standardized phantom filled with a known RC (in both spheres and background) at levels that match clinical conditions. Data were obtained at 4 different 
target-to-background $\mathrm{RC}$ ratios. The phantom was imaged at rest and then moving in 2 orthogonal planes to simulate respiratory motion. All data were acquired in both 2-dimensional (2D) and 3-dimensional (3D) modes of PET acquisition. The purpose of this study was to compare gated with nongated PET acquisitions using absolute quantification of the RC in a moving phantom simulating respiratory motion. A secondary purpose was to compare the $2 \mathrm{D}$ and $3 \mathrm{D}$ modes of PET acquisition for both gated and nongated conditions.

\section{MATERIALS AND METHODS}

\section{NEMA/IEC Phantom}

A PET image quality phantom, the National Electrical Manufacturers Association (NEMA) 2001 International Electrotechnical Commission (IEC) phantom (Data Spectrum Corp.), was imaged in this study (Fig. 1A). The phantom contains 6 hollow spheres with internal diameters of 10, 13, 17, 22, 28, and $37 \mathrm{~mm}$ inside a water-fillable simulated body cavity (the lung insert was not used). In a series of experiments, all the spheres were filled with ${ }^{18} \mathrm{~F}$ solution that was measured in a dose calibrator and then diluted with water to $100 \mathrm{~mL}$ using a volumetric flask to obtain an RC of $19-62 \mathrm{kBq} / \mathrm{mL}$. The phantom background was filled with water, and then varying amounts of ${ }^{18} \mathrm{~F}$ solution were added to obtain an RC of $0.9-10.2 \mathrm{kBq} / \mathrm{mL}$, yielding sphere-to-background (S/B) ratios of approximately $5,10,15$, and 20 to 1 . Three aliquots each of both sphere and background solutions were also counted in a $\gamma$-well counter to determine the true $S / B$ ratios $(4.8,9.7,16.9$, and 21.2). The values of RC for the spheres and background, and the $\mathrm{S} / \mathrm{B}$ ratios, were chosen to simulate the ranges obtained from clinical conditions based on the tumor concentration of ${ }^{18} \mathrm{~F}-\mathrm{FDG}$ and standardized uptake values (SUVs). These clinical values were measured from PET/CT images of 10 different lung carcinoma patients scanned on the same system (Table 1). A motordriven plastic platform drove the phantom up and down a ramp, yielding oblique motion along the longitudinal axis of the scanner (Fig. 1A). The 3 parameters of motion (direction, displacement, and cycle time) were all chosen to match typical respiratory conditions. Directions of motion were in both the craniocaudal (longitudinal) and the anterior-posterior axes; displacement was 2 $\mathrm{cm}$, and cycle time was $5.8 \mathrm{~s}$.

\section{Imaging}

CT transmission (for attenuation correction) and PET emission scans were performed using a PET/CT scanner (Discovery ST-16; GE Healthcare) with a static and moving phantom. Scans of the moving phantom were acquired with and without gating. The helical CT scan was performed first $(120 \mathrm{kV}$ and $300 \mathrm{~mA})$, and then PET emission data were acquired for $500 \mathrm{~s}$ for 1 field of view. All PET scan types (static, motion nongated, and gated) were acquired in both 2D and 3D modes. The 3D data were first Fourier rebinned, and then all emission scans were reconstructed into a 256 matrix, using ordered-subset expectation maximization algorithms. The number of iterations and subsets was 2 and 30, respectively, for the $2 \mathrm{D}$ mode and 5 and 32 , respectively, for the 3D mode. The PET reconstructed slice thickness was $3.27 \mathrm{~mm}$. For the gated data, only bin number 3 of a total of 10 bins in the cycle was reconstructed.

\section{Motion Gating}

The simulated respiratory motion was detected and recorded using the real-time position management system (Varian Medical Systems). This system uses an infrared camera and reflective markers placed on the moving object (the phantom platform) to track motion (5). The average motion cycle was measured and then divided by 10 to obtain the fixed time bin used for each of the 10 bins of the motion-gated PET scan.

\section{Data Analysis}

The background $\mathrm{RC}$ was determined from the images by using a modified version of the NEMA NU 2-2001 standard image analysis. The method consisted of using 6 circular regions of interest (ROI) of 30-mm diameter placed in standard locations on the best-visualized central slice and 4 other slices at approximately \pm 1 and $\pm 2 \mathrm{~cm}$ (exact values, \pm 0.981 and $\pm 1.962 \mathrm{~cm}$ ) from the central slice. Mean pixel values in all 30 ROIs were obtained to determine an average image-derived RC.

Two methods were used to measure the absolute RC in the images of the spheres. First, the GE computer system (Xeleris; GE Healthcare) was used to place circular ROIs equal to the known sphere sizes on the 4 largest spheres on a single central reconstructed axial slice as determined by visual assessment. Maximum and mean pixel values of $\mathrm{RC}$ were obtained from within the ROIs. Only the 4 largest spheres were used for all data analyses because the 2 smallest spheres (10 and $13 \mathrm{~mm}$ ) underestimated RC even in the standard static scan because of partialvolume effects (Fig. 1B).

The second method used a radiation therapy planning system (Pinnacle; Philips) to automatically contour volumes of interest with predetermined thresholds relative to the maximum sphere RC on multiple slices for volume quantification. For 2D acquisitions, a threshold of $70 \%$ of the maximum sphere RC was used. This

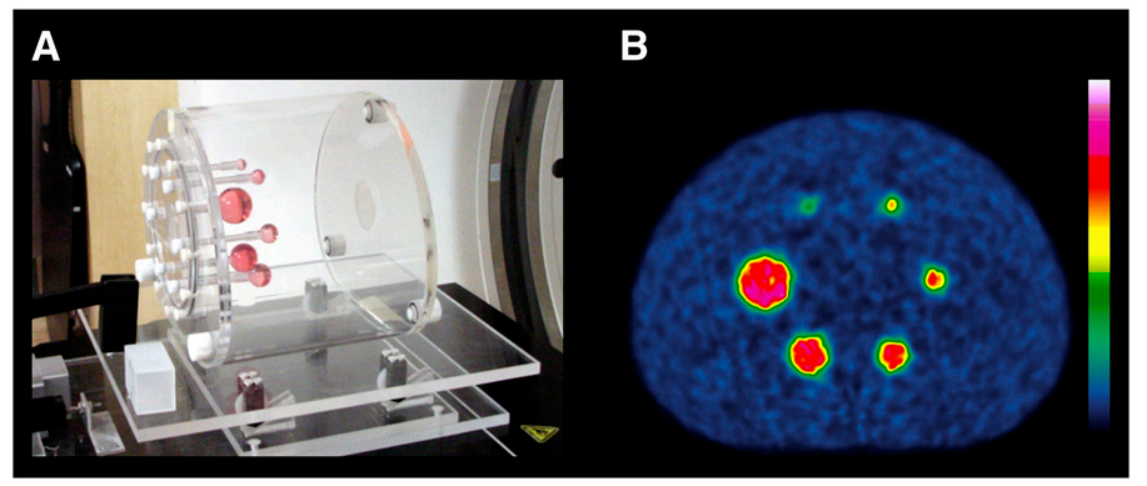

FIGURE 1. (A) NEMA/IEC 2001 phantom (Data Spectrum) on plastic platform. (B) PET mid-transaxial slice through static acquisition in 2D mode with 9.7 S/B ratio. 
TABLE 1

$\mathrm{RC}$, Uptake, and Size

\begin{tabular}{cccc}
\hline Site & $\mathrm{kBq} / \mathrm{mL}$ & SUV or S/B & Centimeters \\
\hline Patient (tumors) & & & \\
Mean & 36.5 & 20.7 & 4.1 \\
$\quad$ Range & $7.4-82$ & $2.9-57$ & $1.7-8$ \\
Phantom (spheres) & & & \\
$\quad$ Mean & 39.1 & 13.2 & 2.1 \\
Range & $19-62$ & $4.8-21$ & $1.0-3.7$ \\
\hline
\end{tabular}

threshold was determined such that the mean $\mathrm{RC}$ in the region within the threshold yielded the expected RC from the 37-mm sphere on a separate experiment using a high RC static scan. Similarly, for 3D acquisitions, a threshold of $94 \%$ was determined and then used.

To compare the image-derived $\mathrm{RC}$ of the background and spheres $\left(\mathrm{RC}_{\mathrm{i}}\right)$ with the expected $\mathrm{RC}$ as measured on the dose calibrator and then decay corrected to scan times $\left(\mathrm{RC}_{\mathrm{e}}\right)$, we calculated percentage differences. These are defined as follows:

$$
\% \text { difference }=\left(\frac{\mathrm{RCi}-\mathrm{RCe}}{\mathrm{RCe}}\right) \times 100 \text {. }
$$

For the background $\mathrm{RC}$, correlation coefficients $r^{2}$ were also determined for each scan type to evaluate the correlation between the imaged-derived and expected RCs.

$\mathrm{S} / \mathrm{B}$ ratios were also determined and compared with the true ratios calculated from the $\gamma$-well counter. The $S / B$ ratios are more clinically relevant than absolute values of $\mathrm{RC}$, because $\mathrm{S} / \mathrm{B}$ ratios are directly comparable to the clinically useful SUV term. However, the absolute values of RC are important in that they determine the S/B ratios and, for a phantom study, should be based on clinical conditions. The S/B ratios alone could be within the clinical range, but they do not indicate if the absolute $\mathrm{RC}$ values are also within the range of clinical conditions. To compare all the data, we calculated for each scan type the overall mean percentage difference in image-derived $\mathrm{RC}$ from expected $\mathrm{RC}$ averaged over all 4 sphere sizes and all $4 \mathrm{~S} / \mathrm{B}$ ratios.

\section{RESULTS}

Images of CT and PET slices for all 3 scan types that demonstrate qualitatively the effects of motion blur are displayed in Figure 2. The spheres had an elliptic appearance on CT and nongated PET when the phantom was in motion; this appearance was due to the fact that the phantom was moving in 2 orthogonal directions. The spheres appeared to be less blurred in the gated PET image than in the nongated image and were almost restored to the static condition.

The image-derived absolute RCs in the phantom background were strongly linearly correlated to the expected RCs for all scan types, in both 2D and 3D modes of acquisition, as illustrated in Figure 3. The squared correlation coefficients $r^{2}$ for all conditions had values of more than 0.99 . The absolute values of the mean percentage differences were $1.8,2.0$, and 2.8 in $2 \mathrm{D}$ mode and $0.9,0.6$, and 2.2 in $3 \mathrm{D}$ mode, for static, nongated, and gated acquisitions, respectively.

A comparison of the maximum, mean, and threshold methods of determining sphere RC for all scan types in 2D mode is shown in the 3 panels of Figure 4, which presents data for the image-derived and expected RCs for the largest sphere $(37 \mathrm{~mm})$. The mean pixel method was the only method by which the results for the gated scan showed a linear relationship (Fig. 4B). The underestimation of imagederived RC (using the mean pixel value) is illustrated in the histograms of Figure 5 for the 4 different sphere sizes and $\mathrm{S} / \mathrm{B}$ ratios in both $2 \mathrm{D}$ and $3 \mathrm{D}$ modes. The overall mean percentage differences between image-derived and expected RC for the spheres (all values were underestimated) were 37,49 , and 41 in 2D mode and 40, 51, and 41 in 3D mode for static, nongated, and gated acquisitions, respectively. This result demonstrates that gated acquisitions improved quantification over nongated acquisitions by reducing the underestimation by $8 \%$ (from $49 \%$ to $41 \%$ ) and $10 \%$ (from $51 \%$ to $41 \%$ ), respectively, for $2 \mathrm{D}$ and $3 \mathrm{D}$ modes of PET acquisition.
FIGURE 2. (A and B) Sagittal images acquired through 13- and 22-mm spheres with phantom at rest in 2D mode and with 9.7 S/B ratio: CT (A) and PET (B). (C-E) Images acquired with same phantom in motion: CT (C), nongated PET (D), and gated ( 1 of 10 time bins) PET (E). All PET images have been scaled relative to each other. On CT images, air in stems of spheres appears black.





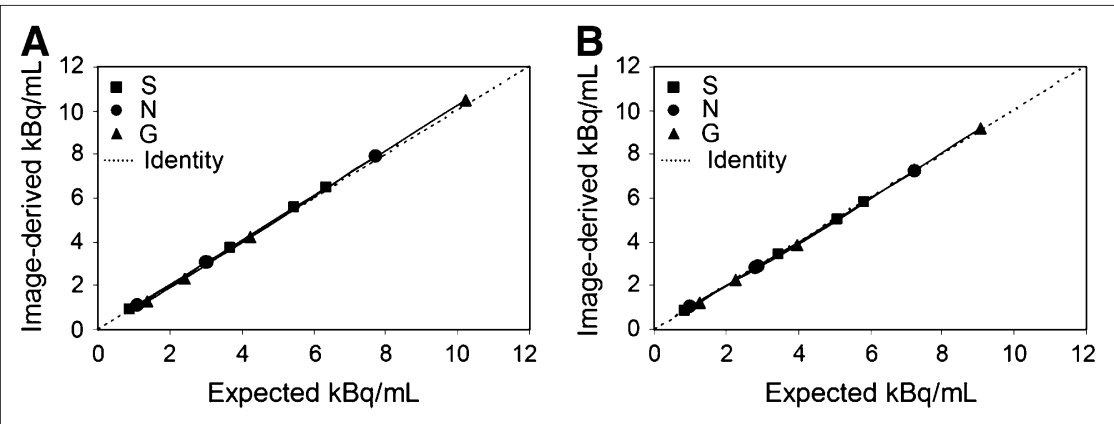

FIGURE 3. Image-derived and expected $R C$ in phantom background, in 2D (A) and 3D (B) modes of PET acquisition. The 3 types of scans were static $(\mathrm{S})$, motion nongated $(\mathrm{N})$, and gated $(\mathrm{G})$, and each series was fitted with linear regression.

The relationship between the image-derived and true S/B ratios for the 3 types of PET scans (Fig. 6) was similar to that between the image-derived and expected sphere RC results in Figure 4B.

\section{DISCUSSION}

In this study, a phantom was used because it allowed the expected RC to be measured without motion and then in the presence of quantified motion, and because it allowed the effects of gated acquisitions to be compared with the effects of nongated acquisitions and with the gold standard static acquisition.

All experiments in this study were conducted over approximately $8 \mathrm{mo}$. During that time, the PET scanner was cross-calibrated to the dose calibrator 3 separate times as part of a routine quality control program. Because of the strong linear correlation found between the image-derived RCs and the expected RCs in both 2D and 3D modes (Fig. 3 ), the cross-calibration factors were accurate throughout the experiments and the scanner was operating with high quantitative accuracy.

Comparison of the methods for determining sphere $\mathrm{RC}$ using the maximum and mean pixel values indicates that maximum pixel values of RC were overestimated and, under the gated condition specifically, did not provide a good estimate of RC (Fig. 4A). The reasons for this overestimation and random behavior are varied and could be related to the reconstruction algorithm, increased noise in the gated images, or both. The noise in the gated images is due to low count statistics $(10,11)$, because each gated time bin is acquired over only one tenth of the time of both the static and nongated acquisitions. Histogram plots of RC in voxels within a volume of interest surrounding the largest sphere for both static and gated acquisitions (Fig. 7) demonstrate an increased number of voxels with $\mathrm{RC}$ values that are above the expected value in the gated acquisition (Fig. 7B). These voxels contain increased noise (due to the low statistics), and therefore the maximum pixel method was not used. Using the mean pixel method, RCs for all scan types were underestimated, compared with the expected values (Fig. 7); however, all scan types demonstrated linear responses (Fig. 4B).

The threshold method of measuring sphere RCs was successful in both static and nongated acquisitions, because these are almost equal to the line of identity (Fig. 4C), but again in the gated acquisition this method was of limited use. To directly compare all 3 scan types, the method of quantification should demonstrate a "predictable" response for all 3 scans - that is, a response not affected by noise.

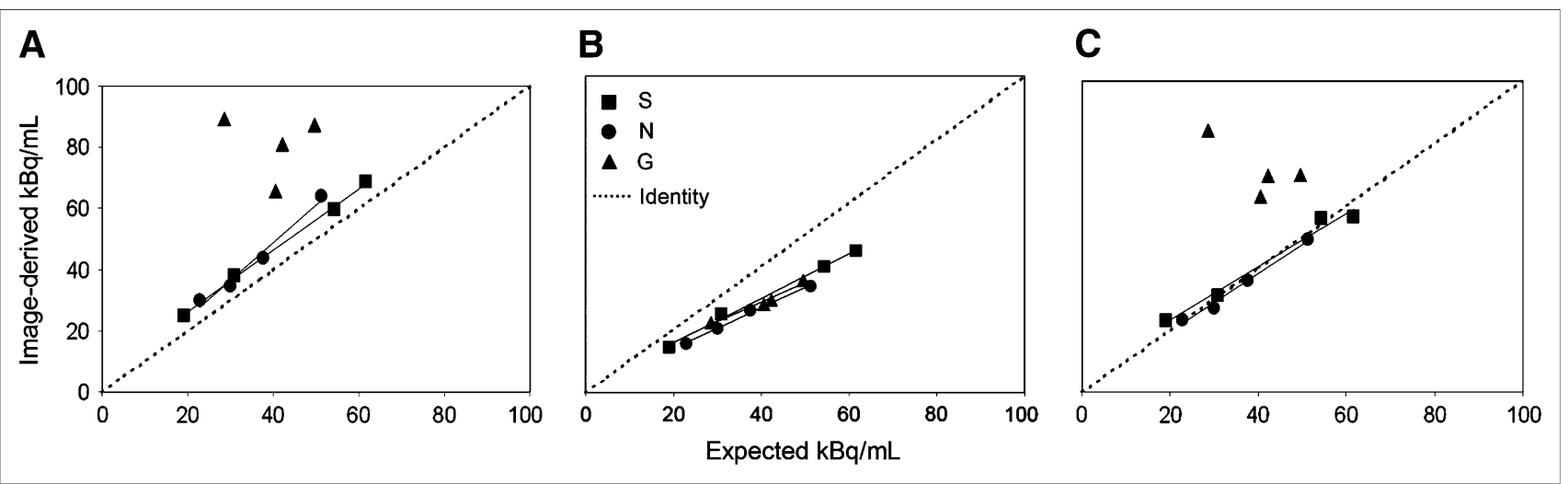

FIGURE 4. Comparison of image-derived and expected sphere RC for data analysis methods: maximum pixel (A), mean pixel (B), and threshold $(\mathrm{C})$. Data are for largest sphere $(37 \mathrm{~mm})$ in 2D mode of PET acquisition for each scan type-static (S), motion nongated $(\mathrm{N})$, and gated $(\mathrm{G})$ — and each series was fitted with linear regression. 


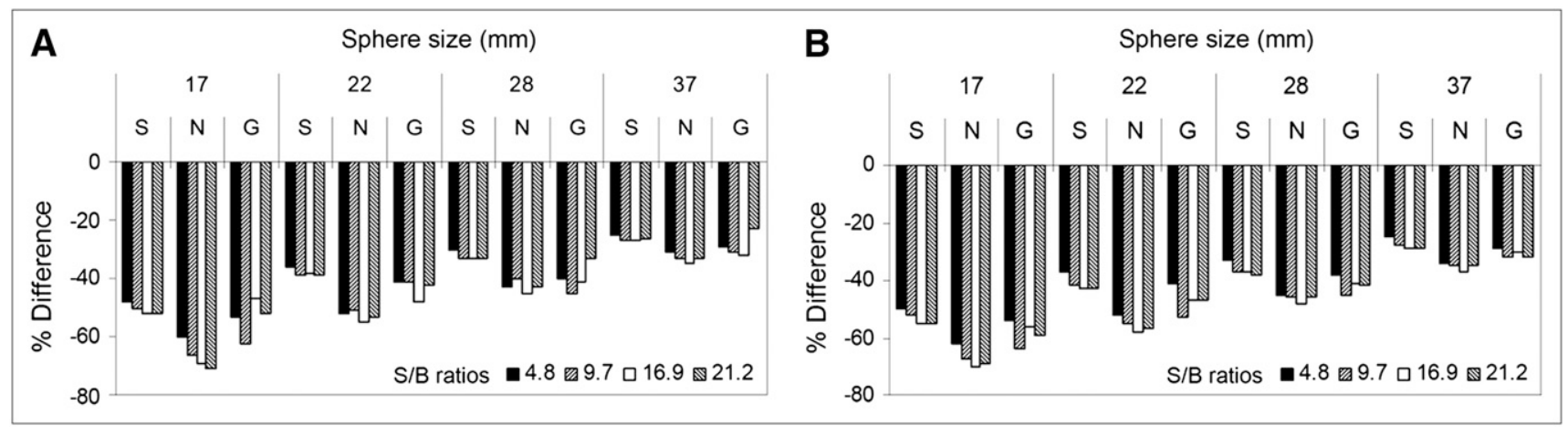

FIGURE 5. Underestimation of image-derived $R C$ from expected value, in 2 modes of PET acquisition: 2D (A) and 3D (B). Data are shown for all sphere sizes $(17,22,28$, and $37 \mathrm{~mm}), \mathrm{S} / \mathrm{B}$ ratios $(4.8,9.7,16.9$, and 21.2), and scan types (static [S], motion nongated $[\mathrm{N}]$, and gated $[\mathrm{G}])$.

Thus, the mean pixel method is the only method enabling direct comparisons. Using this method, all 4 sphere sizes in the 4 different $\mathrm{S} / \mathrm{B}$ ratios showed that gated acquisition reduced the underestimation and demonstrated improvement in absolute quantification of $\mathrm{RC}$, as compared with the nongated acquisition (Fig. 5). This same trend was observed in both 2D and 3D modes of PET acquisitions and also demonstrated that the underestimation was directly related to sphere size (Fig. 5).

Use of the mean pixel method, the S/B ratios, which are analogous to SUV, also demonstrated that gating improved quantification at each S/B ratio over that for nongated acquisitions in both 2D and 3D modes (Fig. 6).

The secondary purpose of this study, to compare $2 \mathrm{D}$ and 3D modes of gated acquisition, showed little difference in improvement ( $8 \%$ and $10 \%$, respectively) using the mean pixel method of quantification. It is, however, difficult to directly compare 2D and 3D modes of acquisition, because different reconstruction methods and parameters were used. The default parameters as recommended by the manufacturer were used, changing only the matrix size from 128 to 256 to improve ROI resolution by having smaller pixel sizes.

The results of this study are comparable to the results of other published phantom studies showing that motion causes an underestimation of sphere RC that is directly related to the sphere size $(7,8)$. This study goes further by measuring the improvement of quantification using gated acquisitions.
This improvement of quantification using gated acquisitions was found using a phantom with RCs, S/B ratios, and sphere sizes that closely matched those of patients (Table 1), in both 2D and 3D modes. As well, this study evaluated simulated respiratory motion in 2 directions (craniocaudal and anterior-posterior axes) -motion that is closer to clinical conditions than motion in only 1 direction (9).

In this study, we observed, as have others (10-13), the limitation of increased noise when using gated acquisition. Various new methods have been proposed to reduce the noise, such as the use of reconstruction techniques $(10,11)$ or of motion correction before reconstruction $(12,13)$. One possible simple solution to reducing the noise would be to scan for a longer time. However, because this solution is not always practical with patients, another approach could be to decrease the number of bins used for gating while keeping the same acquisition time. For example, if the number of bins were reduced from 10 to 6 , there would be 1.7 times more data per bin, thus reducing the noise, at the expense of increased motion blur. In this study, even with the noise from using 10 bins, gated acquisitions were more accurate in quantification than nongated.

Another possible limitation of gated PET for clinical use is that respiratory motion in patients is assumed to be regular; this is not always the case. However, list mode gated acquisitions have been suggested as a solution for patients with irregular respiratory motion cycles because
FIGURE 6. Relationship between imagederived and true $S / B$ ratios in $2 \mathrm{D}(\mathrm{A})$ and 3D (B) modes of PET acquisition. Data are shown for 2 sphere sizes (17 and 37 $\mathrm{mm}$ ) and all scan types (static [S], motion nongated $[\mathrm{N}]$, and gated $[\mathrm{G}]$ ), and each series was fitted with linear regression.
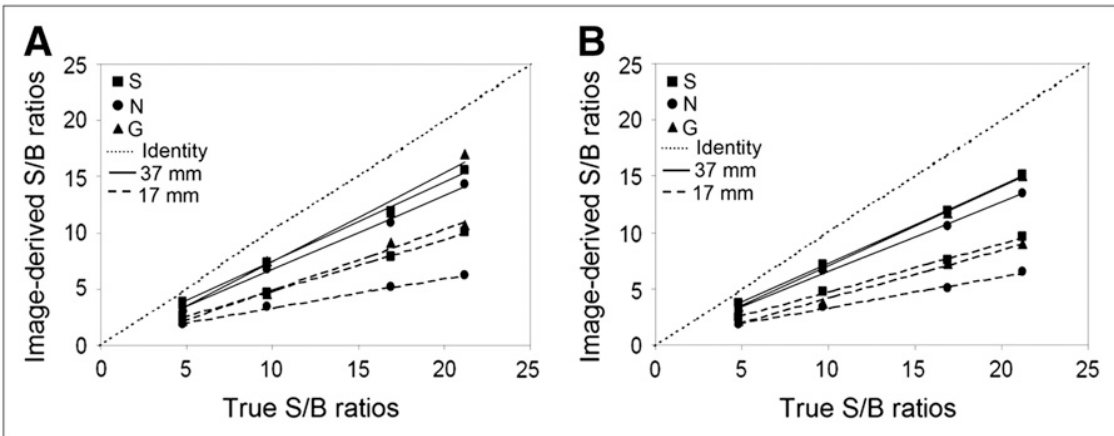


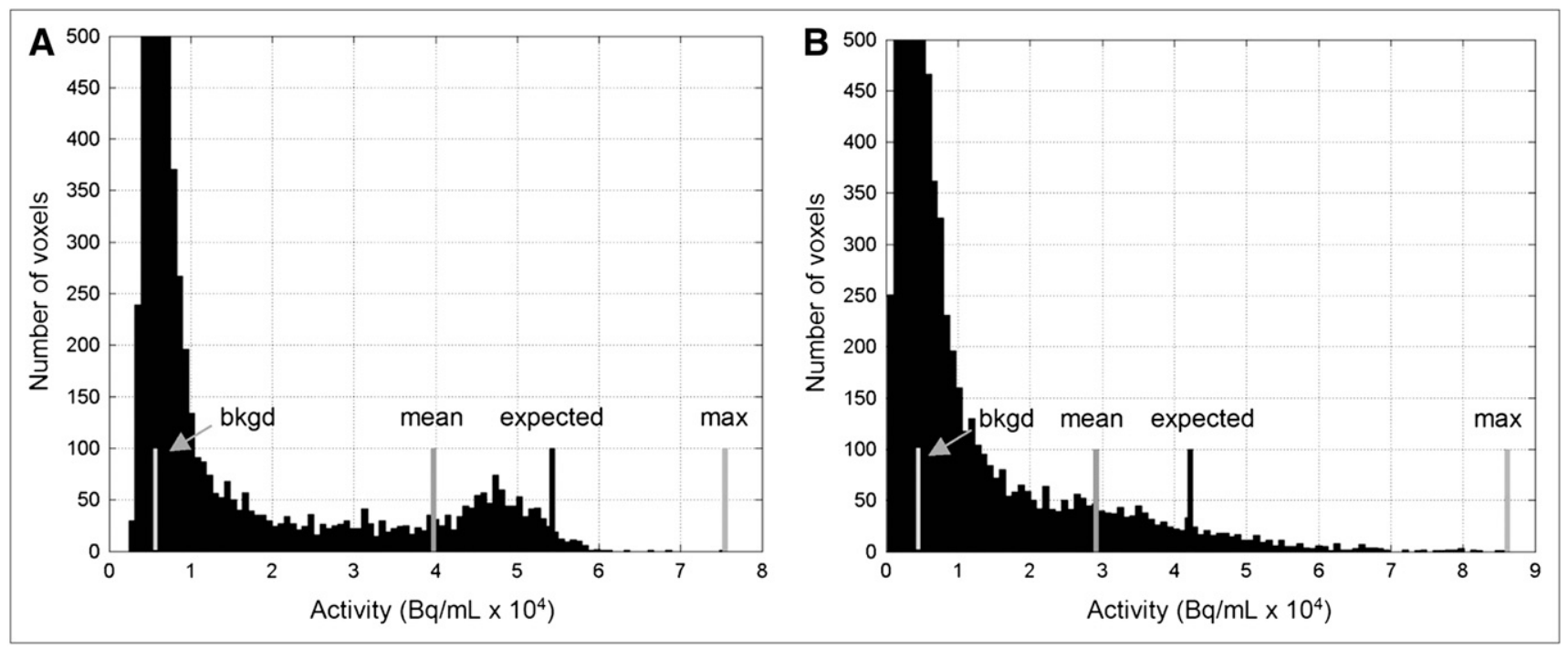

FIGURE 7. Relationship between number of voxels and RC in static (A) and gated (B) scan types. Data are from $56-\mathrm{mm}$ volume of interest around largest sphere $(37 \mathrm{~mm})$ in S/B ratio of 9.7 and in 2D mode of PET acquisition. Indicated on histograms are measured maximum, mean, and background values of $\mathrm{RC}$ from within volume of interest; also marked is expected $\mathrm{RC}$ in sphere.

the list mode data can be rebinned accordingly to remove the irregular cycles (14).

The clinical implication from this phantom study is that the use of gated acquisition in the presence of respiratory motion can improve quantification of PET data. This improvement in PET quantification may potentially lead to improvement in the ability to stage disease in patients and monitor the effects of treatment response.

\section{CONCLUSION}

In the presence of motion, gated acquisitions improved quantification over nongated acquisitions, almost restoring the results to those observed when the phantom is static. There appeared to be little difference between 2D and 3D modes of gated PET when the default parameters of reconstruction and the mean pixel values for quantification were used. Thus, both $2 \mathrm{D}$ and $3 \mathrm{D}$ modes of gated PET improved the absolute quantification of $\mathrm{RC}$ in a moving NEMA phantom.

\section{REFERENCES}

1. Hoekstra CJ, Paglianiti I, Hoekstra OS, et al. Monitoring response to therapy in cancer using $\left[{ }^{18} \mathrm{~F}\right]$-2-fluoro-2-deoxy-D-glucose and positron emission tomography: an overview of different analytical methods. Eur J Nucl Med. 2000;27:731743.
2. Weber WA. Use of PET for monitoring cancer therapy and for predicting outcome. J Nucl Med. 2005;46:983-995.

3. Lammertsma AA, Hoekstra CJ, Giaccone G, Hoekstra OS. How should we analyse FDG PET studies for monitoring tumour response? Eur J Nucl Med Mol Imaging. 2006;33(suppl 13):16-21.

4. Weber WA, Figlin R. Monitoring cancer treatment with PET/CT: does it make a difference? J Nucl Med. 2007;48(suppl 1):36S-44S.

5. Nehmeh SA, Erdi YE, Ling CC, et al. Effect of respiratory gating on reducing lung motion artifacts in PET imaging of lung cancer. Med Phys. 2002;29:366371.

6. Nehmeh SA, Erdi YE, Ling CC, et al. Effect of respiratory gating on quantifying PET images of lung cancer. $J$ Nucl Med. 2002;43:876-881.

7. Boucher L, Rodrigue S, Lecomte R, Benard F. Respiratory gating for 3-dimensional PET of the thorax: feasibility and initial results. J Nucl Med. 2004;45:214-219.

8. Pevsner A, Nehmeh SA, Humm JL, Mageras GS, Erdi YE. Effect of motion on tracer activity determination in CT attenuation corrected PET images: a lung phantom study. Med Phys. 2005;32:2358-2362.

9. Wolthaus JW, van Herk M, Muller SH, et al. Fusion of respiration-correlated PET and CT scans: correlated lung tumour motion in anatomical and functional scans. Phys Med Biol. 2005;50:1569-1583.

10. Li T, Thorndyke B, Schreibmann E, Yang Y, Xing L. Model-based image reconstruction for four-dimensional PET. Med Phys. 2006;33:1288-1298.

11. Qiao F, Pan T, Clark JW Jr, Mawlawi OR. A motion-incorporated reconstruction method for gated PET studies. Phys Med Biol. 2006;51:3769-3783.

12. Thorndyke B, Schreibmann E, Koong A, Xing L. Reducing respiratory motion artifacts in positron emission tomography through retrospective stacking. Med Phys. 2006;33:2632-2641.

13. El Naqa I, Low DA, Bradley JD, Vicic M, Deasy JO. Deblurring of breathing motion artifacts in thoracic PET images by deconvolution methods. Med Phys. 2006;33:3587-3600.

14. Bailey DL, Kalemis A. Externally triggered gating of nuclear medicine acquisitions: a useful method for partitioning data. Phys Med Biol. 2005;50: N55-N62. 